
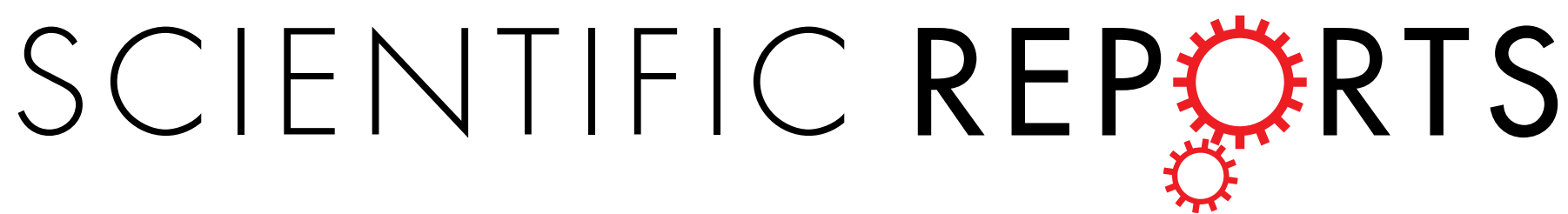

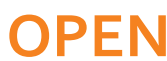

Received: 24 November 2014

Accepted: 27 March 2015 Published: 15 May 2015

\section{Morphological Characterisation of Unstained and Intact Tissue Micro- architecture by X-ray Computed Micro- and Nano-Tomography}

Lucy A. Walton ${ }^{1, *}$, Robert S. Bradley ${ }^{2, *}$, Philip J. Withers ${ }^{2}$, Victoria L. Newton ${ }^{3}$, Rachel E. B. Watson ${ }^{3}$, Clare Austin ${ }^{1,4, *}$ \& Michael J. Sherratt ${ }^{3, *}$

Characterisation and quantification of tissue structures is limited by sectioning-induced artefacts and by the difficulties of visualising and segmenting $3^{D}$ volumes. Here we demonstrate that, even in the absence of X-ray contrast agents, X-ray computed microtomography (microCT) and nanotomography (nanoCT) can circumvent these problems by rapidly resolving compositionally discrete $3^{D}$ tissue regions (such as the collagen-rich adventitia and elastin-rich lamellae in intact rat arteries) which in turn can be segmented due to their different $X$-ray opacities and morphologies. We then establish, using X-ray tomograms of both unpressurised and pressurised arteries that intra-luminal pressure not only increases lumen cross-sectional area and straightens medial elastic lamellae but also induces profound remodelling of the adventitial layer. Finally we apply microCT to another human organ (skin) to visualise the cell-rich epidermis and extracellular matrix-rich dermis and to show that conventional histological and immunohistochemical staining protocols are compatible with prior X-ray exposure. As a consequence we suggest that microCT could be combined with optical microscopy to characterise the $3 \mathrm{D}$ structure and composition of archival paraffin embedded biological materials and of mechanically stressed dynamic tissues such as the heart, lungs and tendons.

Characterising tissue sub-structure by conventional histological sectioning (microtomy) frequently induces tears, fractures, compressions and folds ${ }^{1}$ and, in the specific case of large arteries, often causes detachment of the adventitial layer. Crucially, in order to accurately characterise the structure of tissues such as elastic arteries, it will also be necessary to image sufficiently large regions of the vessel in three-dimensions $(3 \mathrm{D})^{2,3}$. Although recent advances in serial block face scanning electron microscopy and confocal laser microscopy have been employed to visualise the $3 \mathrm{D}$ structure of the aortic medial layer $^{3}$ and adventitial collagen ${ }^{4}$, these techniques can only be applied to relatively small tissue volumes. However, X-ray computed microtomography (microCT) which is conventionally used for determining the structure of hard calcified tissues such as bone, can also be used to visualise the 3D morphology of intact non-calcified organs and organisms in the presence of X-ray contrast agents ${ }^{5,6}$. In this study we aimed to determine if sub- $\mu \mathrm{m}$ tissue-structures (in large arteries and skin) could be visualised in the absence of exogenous X-ray contrast agents.

Large conduit arteries protect smaller vessels from damaging variations in blood pressure ${ }^{7}$. This mechanical dampening function is mediated primarily by cross-linked elastin in the medial elastic lamellae and by collagen fibrils in the outer adventitial layer ${ }^{8}$. Aberrations in this wall structure, as a consequence of heritable connective tissue disorders, ageing and diseases such as diabetes, are associated with

${ }^{1}$ Institute of Cardiovascular Sciences. ${ }^{2}$ School of Materials. ${ }^{3}$ nstitute of Inflammation and Repair, University of Manchester, Manchester, United Kingdom. "Faculty of Health and Social Care, Edge Hill University, Ormskirk, United Kingdom. ${ }^{*}$ These authors contributed equally to this work. Correspondence and requests for materials should be addressed to M.J.S. (email: michael.sherratt@manchester.ac.uk) 


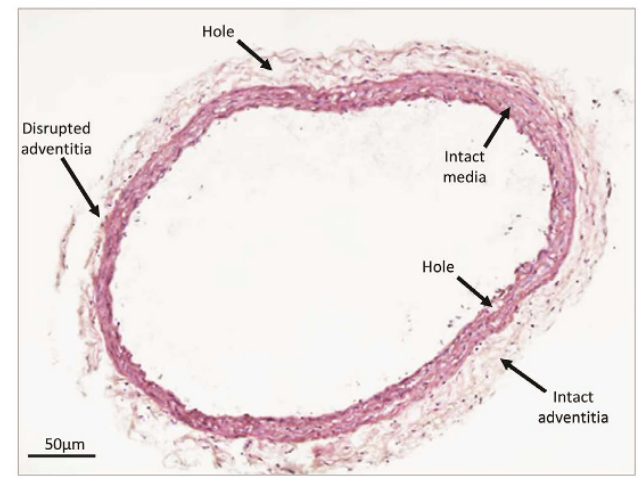

Figure 1. Sectioning-induced artefacts in paraffin embedded vascular and cutaneous tissues. Light microscopy image of $\mathrm{H} \& \mathrm{E}$ stained rat CCA.

life-threatening cardiovascular events including heart failure and aortic aneurysm ${ }^{9-11}$. In order therefore to characterise both the pathological progression of these disorders and the efficacy of potential treatments it is important to visualise arterial wall structure. In practice this is commonly achieved by two dimensional sectioning of unpressurised vessels and subsequent characterisation of the medial layer only. In pioneering work published in 1964, Wolinsky and Glagov documented acute reductions in elastic lamellae "waviness" and medial layer thickness with increasing intra-luminal pressure ${ }^{12}$. In their model system, the New Zealand White Rabbit, the majority of these acute tissue remodelling events took place at sub-physiological pressures ${ }^{13}$ of less than $80 \mathrm{~mm} \mathrm{Hg}$. Despite this clear evidence that the structure of the pressurised vessel differs profoundly from the structure of the unpressurised vessel (a state which only occurs after death) and that the outer adventitial layer also plays a vital role in limiting vessel distension $^{14}$ and preventing aneurysms $s^{15}$, with few exceptions ${ }^{3,4,16,17}$ arterial morphology is still routinely characterised in $2 \mathrm{D}$ from sectioned, unpressurised, tissues.

Here, we characterise the structure of intact Wistar rat large arteries (preserved in either an unpressurised or pressurised state) using microCT. We demonstrate that this technique can differentiate between sub-tissue level layers and that intra-luminal pressure results in profound remodelling of both the medial and adventitial layers. In future studies, it will be important to progress from the static application of intra-luminal pressure (in which water flow may influence tissue architecture and volume over long time scales) to dynamic $\mathrm{x}$-ray imaging of native arteries subjected to physiological cyclic loading regimes. We also demonstrate that X-ray micro-tomography is applicable to other organs such as human skin and that sufficient contrast can be achieved without the use of X-ray contrast agents (which can interfere with subsequent histological and immunohistochemical analyses). As a consequence the technique will be applicable to most archival paraffin embedded biological material.

\section{Results and discussion}

Microtomography Preserves Arterial Structure and Resolves Compositionally Distinct Tissue Regions and Components. Histological analysis of tissue structure is most commonly performed on thin $(<10 \mu \mathrm{m})$ sections cut from formalin fixed and paraffin embedded samples ${ }^{18}$. Although the formalin-induced amine cross-links and paraffin wax act in concert to chemically strengthen and physically support the tissue ${ }^{19}$, the sectioning process invariably disrupts the structure of organs such as large arteries. As a consequence vascular tissue sections are commonly characterised by folds, holes (primarily, although not exclusively, located in the adventitia) and by separation of the collagen-rich adventitia from the medial external elastic lamina (Fig. 1). By avoiding mechanical sectioning, X-ray microCT is capable of preserving the 3D structure of whole organisms and organs $\mathrm{s}^{5,6}$. Here we show that X-ray microCT can also visualise structurally intact and compositionally distinct regions and macro-molecular assemblies in blood vessels at an approximate spatial resolution of $1.2 \mu \mathrm{m}$ (Fig. $2 \mathrm{~A}, \mathrm{~B}$ ) when using a $4 \mathrm{x}$ objective lens or $0.7 \mu \mathrm{m}$ when using a higher power $20 \mathrm{x}$ lens (Fig. 2C,D). Due to the highly organised architecture of large arteries we can show that common tissue components, the fibrillar collagen-rich adventitia, elastin-rich elastic lamellae and cell-rich inter-lamellar regions, differ in their relative X-ray opacities and can therefore be visualised by this technique. Although in isolation, individual virtual X-ray tomogram slices (such as those depicted in Fig. 2A-D) contain a wealth of structural information, each slice represents only a fraction of the 3D X-ray density data which is encoded in the complete reconstruction. By viewing the 3D data as sequential 2D projections in an animated Video (Video S1) new structural information, such as the topography of the luminal surface, becomes clear. Crucially, although perturbations in the roughness of this surface are thought to play an important role in promoting localised vascular damage (and hence cardiovascular diseases such as atherosclerosis ${ }^{20,21}$ ), conventional sectioning methods are unable to visualise the $2 \mathrm{D}$ micro-topography of the luminal intima/blood interface. 
A

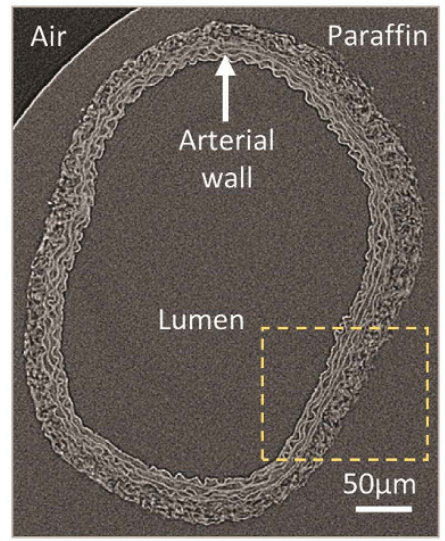

C

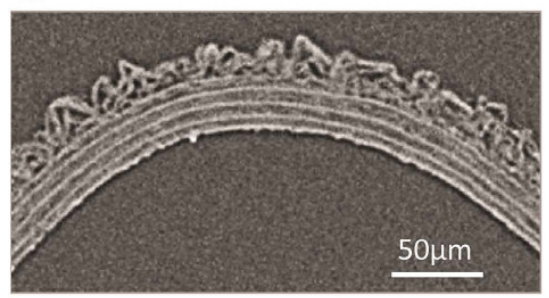

$E$

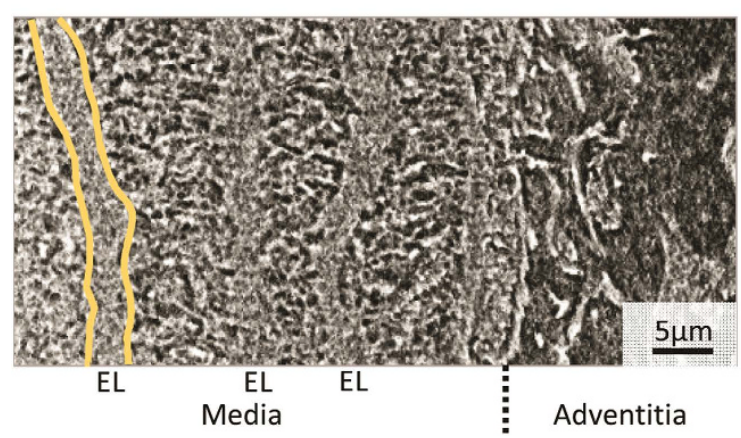

B

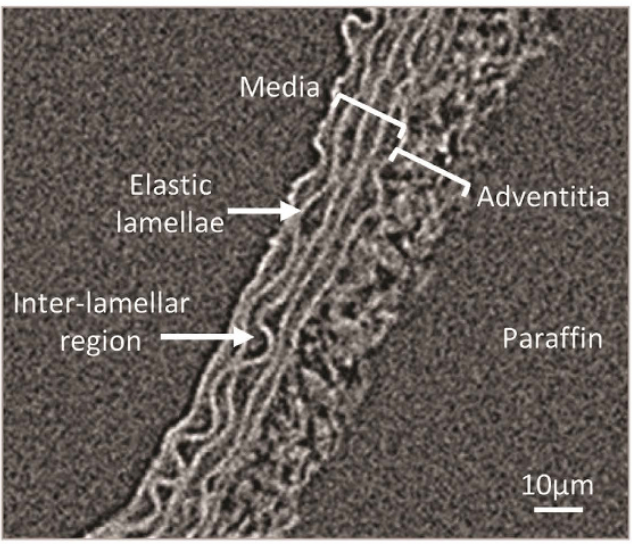

D

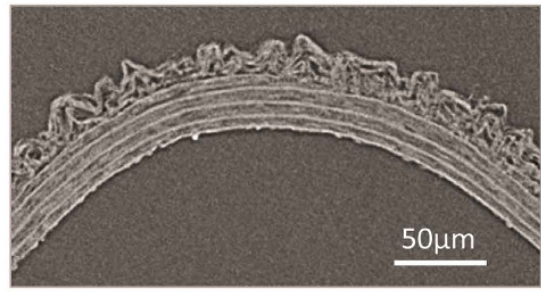

F

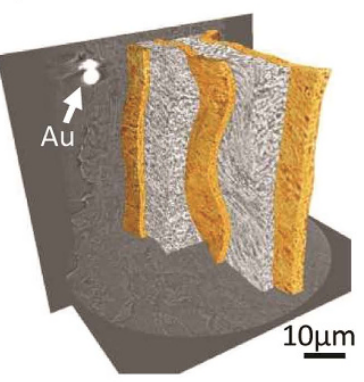

Figure 2. Sub-micron $\mathrm{X}$-ray tomography resolves discrete tissue regions and components in paraffin embedded arteries. (A) Virtual slice extracted from an X-ray tomogram of an intact rat CCA (yellow box indicates magnified region in panel B). Even in the absence of exogenous X-ray contrast agents, the X-ray density of native soft tissues is higher than that of both paraffin and air. (B) Major arterial sub-structures are readily identifiable in the vessel wall. $(\mathbf{C}$ and $\mathbf{D})$ The resolution achievable with a $4 \mathrm{x}$ objective lens $(\mathbf{C}$ : voxel size $0.75 \mu \mathrm{m}, 2 \mathrm{D}$ resolution $1.2 \mu \mathrm{m}$ ) can be improved using a $20 \mathrm{x}$ objective (D: voxel size $0.50 \mu \mathrm{m}, 2 \mathrm{D}$ resolution $0.7 \mu \mathrm{m})$. (E) Virtual slice extracted from an X-ray tomogram at nanoscale resolution $(150 \mathrm{~nm})$ of an intact rat aorta. The tomogram was taken with Zernike phase contrast which gives contrast to edge features and resolves fibrous structures within the inter-lamellar regions. (F) Elastic lamellae and interlamellar regions can be readily segmented and gold particles $(\mathrm{Au})$ can be used to locate the same tissue region on differing instruments.

Until recently, x-ray tomography has been limited in its ability to reveal features within non-calcified, and hence weakly $\mathrm{x}$-ray absorbing, tissues. Whilst $\mathrm{x}$-ray absorption and therefore contrast can be enhanced using high $\mathrm{Z}$ elements ${ }^{22}$ difficulties remain with ensuring that these agents: i) penetrate larger tissues, ii) differentiate between tissue components and iii) are compatible with subsequent histological techniques ${ }^{23}$. Alternatively, $\mathrm{x}$-ray phase contrast approaches can enhance contrast in non-calcified tissues including cartilage ${ }^{24}$, tendon ${ }^{25}$, plaque containing blood vessels ${ }^{26}$, coronary artery ${ }^{27}$, ligaments and the intervertebral $\operatorname{disc}^{28}$. However, such approaches may require complex imaging set-ups ${ }^{29}$ and post-processing ${ }^{30}$, and image acquisition times can be long for laboratory instruments. In this paper, we demonstrate that excellent differential contrast can be achieved in non-calcified tissues with a laboratory microCT system using absorption contrast in conjunction with phase contrast enhancement. We attribute this improved contrast to both our specimen preparation techniques and the instrumentation. Tissue 
samples were prepared using a standard histological procedure which involves chemical fixation, alcohol dehydration and infiltration with paraffin wax. Differential dehydration of tissue components (such as low-density, hydrated cells and densely packed hydrophobic elastic lamellae in large arteries) in combination with localised paraffin infiltration may increase internal contrast. Instrumental improvements include the use of thin scintillators which absorb fewer higher energy x-rays than conventional flat panel detectors $^{31}$. These high energy $\mathrm{x}$-rays do not contribute significantly to contrast of low $\mathrm{Z}$ materials but rather to the background noise. Furthermore, flat panel detectors may contain an Al filter which would further reduce the relative contribution from low energy $\mathrm{x}$-rays to image formation, making them suitable for $\mathrm{x}$-ray energies greater than $\sim 40 \mathrm{keV}^{32}$. Finally, in-line phase contrast enhances contrast at the multiple boundaries (such as lumen-wall and elastic lamellae-interlamellar regions) which are found in tissues such as arteries thereby providing additional edge-enhancement ${ }^{30}$.

The Complex Architecture of Medial Layer Interlamellar Spaces is Evident in Nanotomography Reconstructions. Whilst the X-ray micro-tomograms depicted in panels A-D of Fig. 2 are highly informative, most organs and tissue are organised at multiple length scales. In order therefore to resolve, for example, fine elastic fibres, collagen fibril bundles and vascular smooth muscle cell structure within the arterial medial lamellar unit ${ }^{3,33}$ it is necessary to improve on the $0.5 \mu \mathrm{m}$ resolution which can be achieved by using the Carl Zeiss XRM Xradia Versa microCT. Sub-100 nm resolutions can be achieved with both synchrotron and laboratory $\mathrm{X}$-ray sources ${ }^{34}$ and by using concepts pioneered in optical microscopy (such as Fresnel zone plates) X-rays can be focused with phase contrast achieved by propagation phase contrast or Zernike phase contrast phase rings ${ }^{35}$. These techniques have previously been used to image unstained bacteria, insects and mineralised tissues ${ }^{36,37}$. Here we demonstrate using Zernike phase contrast on a Carl Zeiss XRM Xradia Ultra-810 (with a resolution of $150 \mathrm{~nm}$ ) that, compared with the amorphous appearance which characterises medial interlamellar regions in micro-tomograms, these same areas appear structurally heterogeneous by nanotomography (nanoCT) (Fig. 2B,D). This dense fibrous network may be composed of discrete, small-diameter, elastic fibres and collagen fibril bundles $^{3,33}$. If necessary, tissue structure could be studied across multiple length scales by labelling a region of interest with gold spheres (as depicted by the area of bright contrast in Fig. 2F) hence enabling cross-correlative tomographic imaging of the same sample region by microCT and nanoCT. In order to fully exploit the wealth of $3 \mathrm{D}$ structural data which is present in both this nanoCT reconstruction and microCT data sets, we have developed semi-automatic image analysis approaches to segment structurally and compositionally distinct, yet low-contrast, tissue regions.

Tissue-Specific Segmentation Strategies are Required to Quantitatively Characterise $3 D$ tissue Structure. Large arteries are highly structured tissues in which the major ECM components, elastin and fibrillar collagens, are both X-ray opaque (compared with the paraffin support) and largely confined to the medial elastic lamellae and adventitial layers respectively (Fig. 1,2). Additionally, the elastic lamellae confer an ordered structure on the medial layer which contrasts with the less-ordered adventitial layer. We have developed a sequential image processing protocol which exploits differences in X-ray opacity in order to segment the vessel wall from the supporting paraffin ${ }^{38,39}$ and then uses the morphological characteristics ${ }^{40}$ of the elastic lamellae to segment the media from the adventitia (Fig. 3). By automatically segmenting 3D X-ray tomography volumes, we have been able to: i) avoid potential sampling errors and gain insight into variations in morphology by rapidly characterising these morphological parameters at all points around the vessel cross-section in each of the 450 virtual slices (4x objective), ii) characterise the morphology of the intact adventitial layer and iii) assess the effects of intra-luminal pressure on the topography of clinically important interfaces such as that formed between the luminal surface and the blood stream ${ }^{41}$ (Figs. 4 and 5). A similar approach can be used to segment distinct 3D structures within the medial layer of nanoCT reconstructions (Fig. 2E,F). Having established methods for visualising and measuring the 3D volume and topography of discrete tissue regions and surfaces we can use these approaches to quantify the effects of intra-luminal pressure on the structure of large arteries.

MicroCT Preserves Arterial Structure and Allows for the Measurement of Novel Morphological Characteristics. In 1964 Wolinsky and Glagov reported on the effects of intra-luminal pressure on the $2 \mathrm{D}$ architecture of the aortic media ${ }^{12}$. Here we show that, compared with the unpressurised vessel, the pressurised (at $110 \mathrm{~mm} \mathrm{Hg}$ ) rat common carotid artery (CCA) also exhibits significant structural remodelling (Fig. 4). From the segmented volume, it is apparent that both medial ( $0 \mathrm{~mm} \mathrm{Hg:} 29.99 \pm 0.02 \mu \mathrm{m}$, $110 \mathrm{~mm} \mathrm{Hg}: \quad 25.62 \pm 0.69 \mu \mathrm{m}, \mathrm{p}<0.0001)$ and adventitial $(0 \mathrm{mmHg}: 31.12 \pm 0.11 \mu \mathrm{m}, 110 \mathrm{~mm} \mathrm{Hg}$ : $20.55 \pm 0.03 \mu \mathrm{m}, \mathrm{p}<0.0001$ ) layer thickness are significantly lower in the pressurised vessel (Fig. 5A,B). By segmenting the $3 \mathrm{D} \mathrm{X}$-ray tomogram data we can additionally measure the cross-sectional area (CSA) of the lumen and medial and adventitial layers in each of the 450 slices in the tomogram (Fig. 5C-E). Wolinksy and Glagov ${ }^{12}$ reported a pressure-induced non-linear increase in vessel radius and a decrease in medial layer thickness, here we show that intra-luminal pressure is associated with a greater lumen CSA

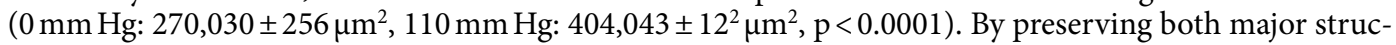
tural layers of the artery we additionally show that the adventitial layer is less uniform in morphology than the medial layer and that the numerous pores (identified by their low X-ray density compared with paraffin wax) which characterise the unpressurised adventitia are considerably reduced in volume and 

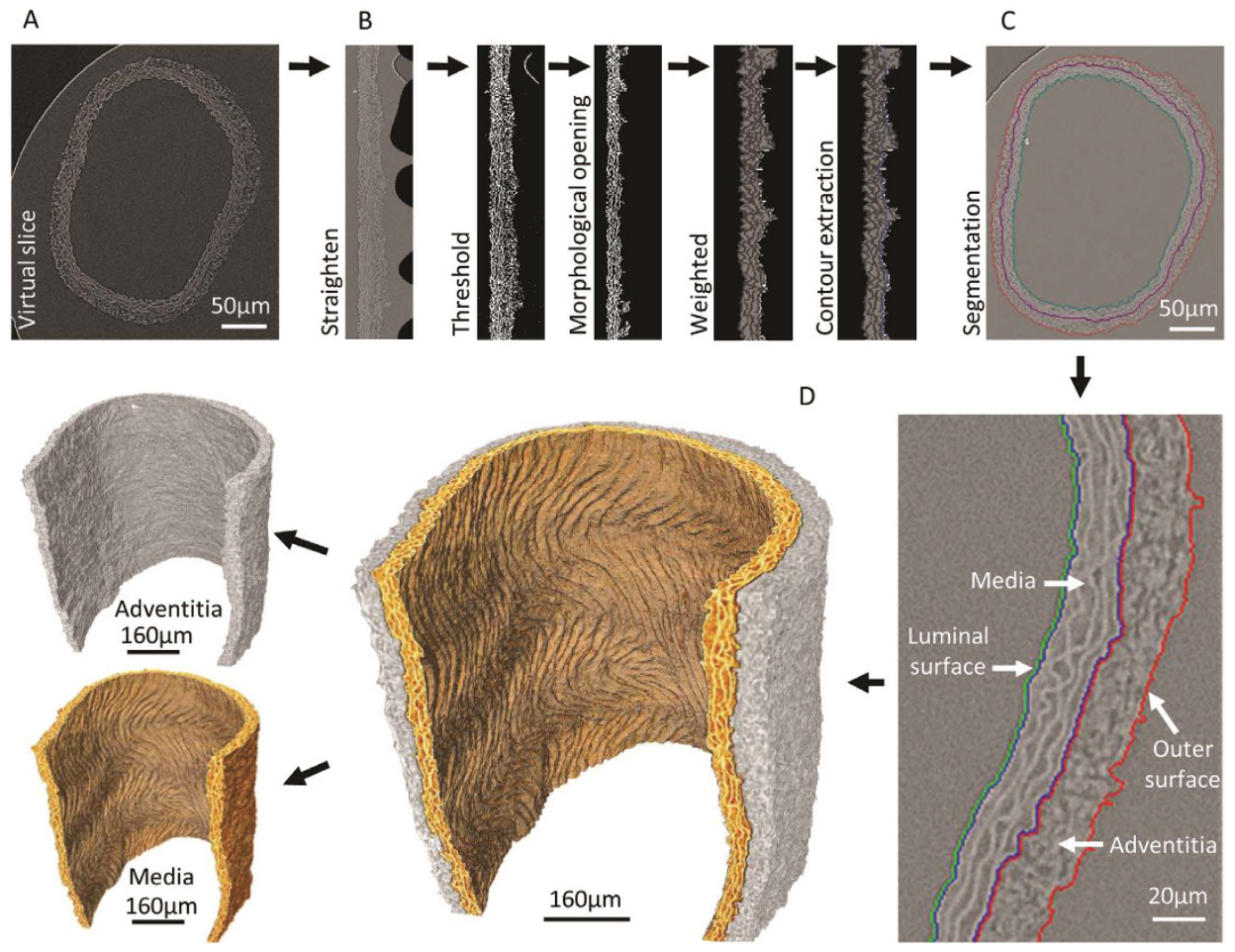

Figure 3. Semi-automatic segmentation of medial and adventitial layers and elastic lamellae in large arteries. (A) Virtual trans-axial slice through an X-ray tomogram of a rat CCA obtained by micro CT. (B) Segmentation of the medial and adventitial layers was performed based on differences in grey level texture using the following steps: i) unwrapping to straighten out the wall, ii) morphological opening and island removal so that only features which are aligned mostly along the vertical direction are retained (most features within the adventitia are removed) and iii) extraction of the contour between the medial and adventitial layers. This is based on an optimization method to find the geodesic path through a weight image. (C) The contour is re-wrapped onto the original slice. (D) Rendering showing the output of the segmentation process which enables the medial and adventitial layers to be virtually dissected.

abundance in the pressurised vessel (Fig. 4E,F). As a consequence of this remodelling, the cross-sectional area (CSA) of the adventitia is significantly reduced in the pressurised vessel (medial CSA: $0 \mathrm{~mm} \mathrm{Hg:}$ 65,258 $\pm 53 \mu \mathrm{m}^{2}, 110 \mathrm{~mm} \mathrm{Hg}: 66,922 \pm 76 \mu \mathrm{m}^{2}, \mathrm{p}<0.0001$; adventitial CSA: $0 \mathrm{~mm} \mathrm{Hg}: 72,658 \pm 228 \mu \mathrm{m}^{2}$, $\left.110 \mathrm{~mm} \mathrm{Hg}: 56,844 \pm 82 \mu \mathrm{m}^{2}, \mathrm{p}<0.0001\right)$. Crucially however, it remains to be determined if comparable adventitial remodelling would be evident either between physiological intra-luminal pressures and/or in dynamic, rather than static, loading conditions. In order to understand the function of healthy and diseased arteries and to design appropriate tissue engineered replacements it will be necessary to model the mechanical behaviour of arterial tissues ${ }^{42}$. MicroCT has the potential to contribute to these models by identifying localised remodelling events in mechanically deformed cardiovascular tissues and in other organs such as aged and hence fragile skin ${ }^{43}$.

MicroCT can Visualise Tissue Sub-Structures in Other ECM-Rich Organs. Skin is an organ which is composed of two main tissue layers: an outer cell-rich and avascular epidermis and a supporting ECM-rich dermis which also contains an extensive microvasculature and discrete structures such as hair follicles. As with large arteries, skin is prone to sectioning induced artefacts (Fig. 6A). Whilst in skin, the epidermis rarely separates from the dermis, both tissue regions are subject to sectioning-induced tears and the separation and delamination of the outer stratum corneum. In contrast, microCT reconstructions clearly show a closely adhered stratum corneum (Fig. 6B,C). This densely packed barrier layer (which is composed primarily of extracellular lipids and disulphide-bonded intracellular keratins), the cellular epidermis, the ECM-rich dermis, hair follicles and potentially a branched micro-vasculature can all be resolved by virtue of their relative X-ray density and architecture (Fig. 6B,C). The 3D architecture of each reconstruction may also be examined by virtual sectioning of the volume at arbitrary angles to visualise, for example, the structure of skin perpendicular (Video S2) and parallel with the surface (Video S3). In this latter video the rhomboidal organisation of the stratum corneum, which is not evident in histological sections, can be clearly seen as X-ray dense circles formed by cross-linked keratin-rich hair shafts. To our knowledge this is the first demonstration that microCT can visualise such skin sub-structures. Previously, 

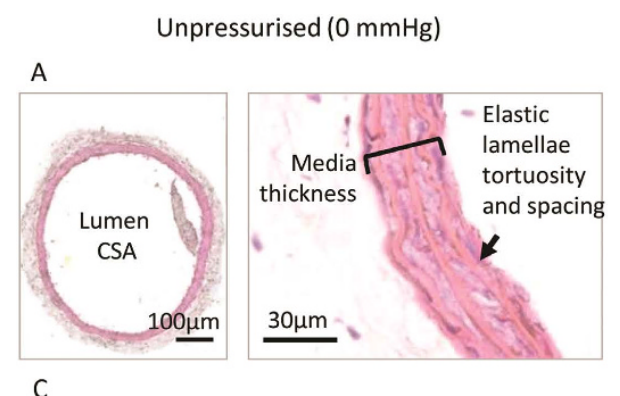

C

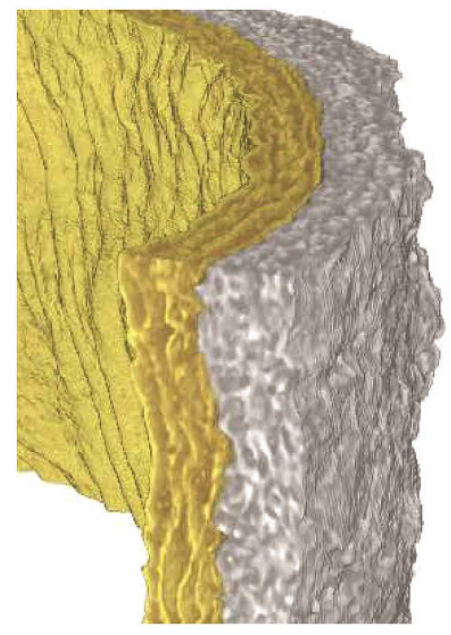

E
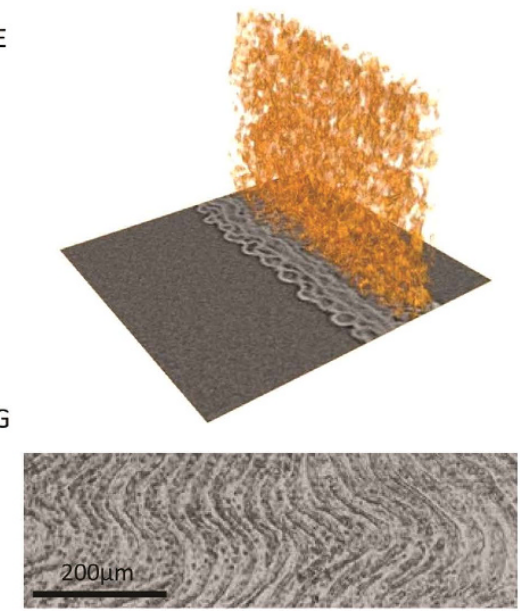

Pressurised $(110 \mathrm{mmHg})$

B

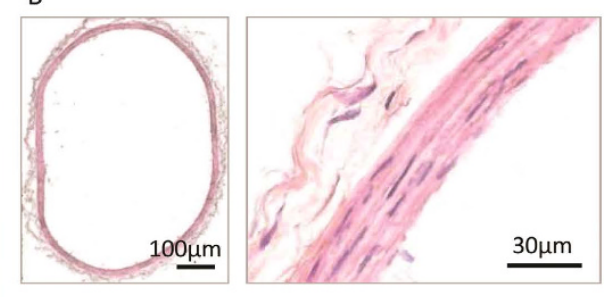

D

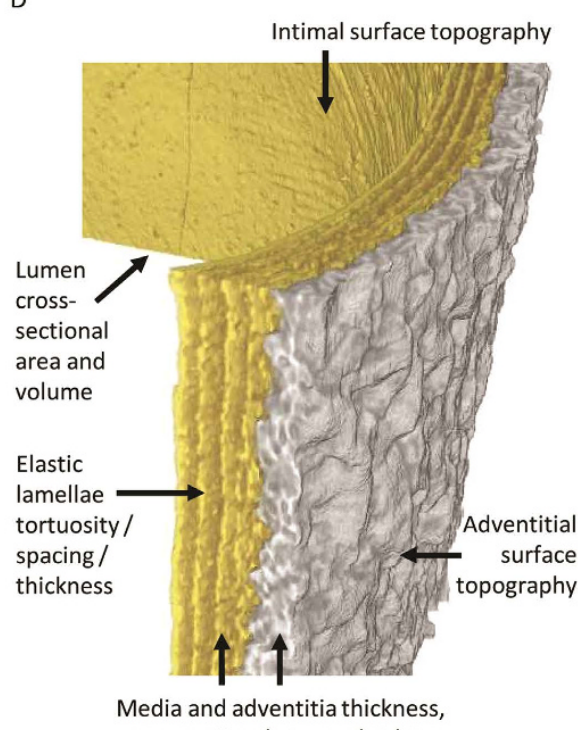

cross-sectional area and volume

$\mathrm{F}$

$\mathrm{H}$
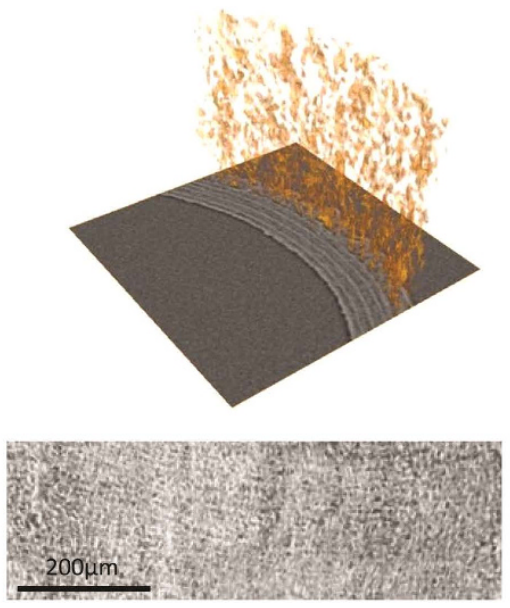

Figure 4. Intra-luminal pressure-induced remodelling of rat common carotid arteries. (A and B) Sections of vessels preserved unpressurised (A) and pressurised (B) states. (C and D) X-ray tomography reconstructions of unpressurised $(\mathbf{C})$ and pressurised $(\mathbf{D})$ vessels. The medial and adventitial layers are segmented in yellow and in grey respectively. (E and F) Segmented pores (with an X-ray density lower than paraffin: depicted as orange 3D volume projections) from unpressurised (E) and pressurised arteries (F) mapped onto a 2D circumferential slice. ( $\mathbf{G}$ and $\mathbf{H}$ ) 3D projections of unwrapped luminal surfaces extracted from reconstructions of unpressurised $(\mathbf{G})$ and pressurised $(\mathbf{H})$ vessels. The unpressurised surface is characterised by a rippled appearance which is oriented along the axis of the vessel.

microCT has been employed to either visualise skin in relation to other organs (whole limb microCT in mice $)^{44}$ or to localise foreign particles such as gunshot residues ${ }^{45}$.

Histological and Immunohistochemical Procedures are Compatible with microCT. It is clear from the data presented in Fig. 2-6 that even in the absence of exogenous X-ray contrast agents X-ray tomography can resolve sub-structures in chemically fixed, paraffin embedded samples. By combining X-ray 

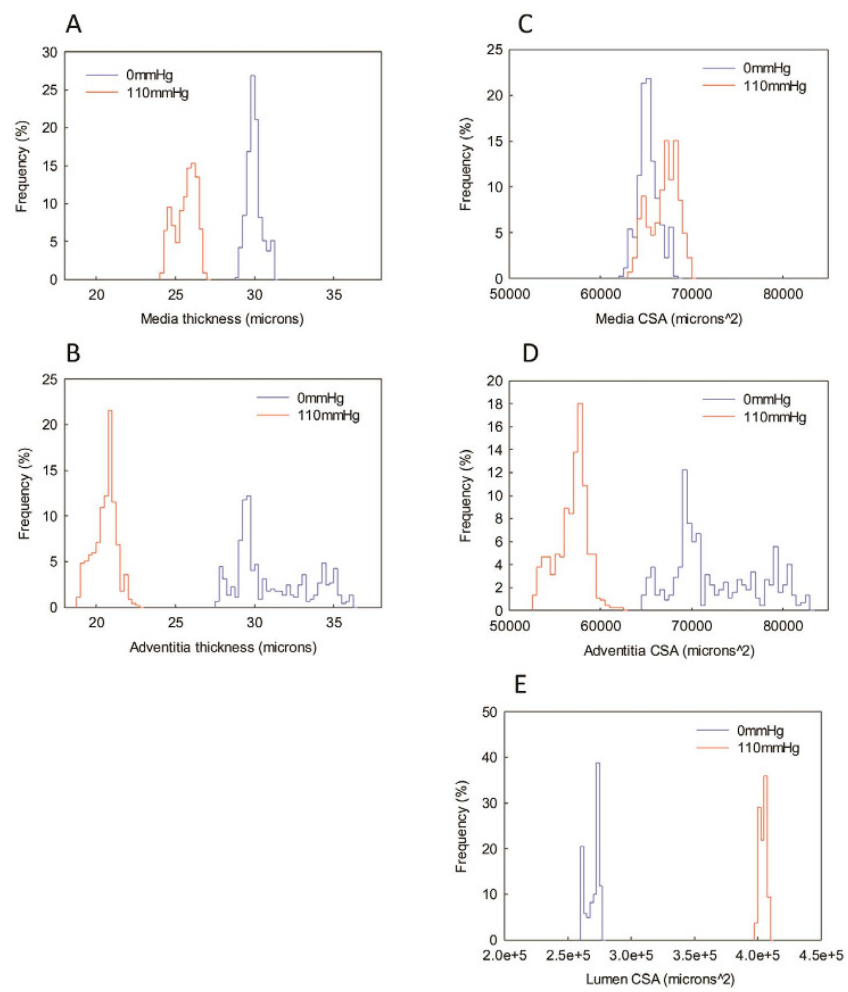

Figure 5. Morphological characterisation of medial and adventitial layer dimensions in unpressurised and pressurised arteries. Frequency distribution histograms of mean medial layer thickness (A) and adventitial layer thickness (B). The cross-sectional area (CSA) of the medial (C) and adventitial (D) layers and the lumen (E) can be rapidly calculated from segmented X-ray tomograms. Thicknesses and areas are derived from a large tomographic volume (450 axial slices, axial length $338 \mu \mathrm{m}$ ).

tomography imaging with subsequent histological ${ }^{46}$ and immunohistochemical analyses it should be possible to confirm the identity of discrete structures in X-ray tomograms. Exposure to synchrotronderived X-rays has been shown to induce thermal and/or oxidative damage to structural protein such as collagen ${ }^{47}$. In this study we examined the effects of X-ray exposure alone (in our microCT system) and $\mathrm{X}$-ray exposure in combination with X-ray contrast agents on the binding of common histological stains to skin sections. Haematoxylin and eosin (H\&E) staining is widely used to distinguish between cellular epidermis and ECM-rich dermis of mammalian skin. Here we used human skin biopsies to characterise: i) the ability of commonly used X-ray contrast agents (phosphotunstic acid [PTA] and inorganic iodine [Lugol's solution; $\mathrm{I}_{2} \mathrm{KI}$ ]) to enhance differentiation of these tissue regions and ii) the effects of $\mathrm{X}$-ray tomography (of both unexposed and PTA- or $\mathrm{I}_{2} \mathrm{KI}$-exposed biopsies) on subsequent H\&E staining. Using both PTA and $\mathrm{I}_{2} \mathrm{KI}$ contrasting agents, good image contrast was achieved between the epidermis and dermis in scans of less than 4.5 hours duration (Fig. 7A). However, as previously reported ${ }^{5}$ tissue $^{2}$ penetration of PTA was slow. Full penetration of a $3 \mathrm{~mm}$ diameter skin biopsy was not achieved after 40 hours exposure (Fig. 7B). Furthermore, whilst PTA exposure inhibited subsequent H\&E staining, both epidermal and dermal cells and the dermal matrix were clearly stained following $\mathrm{X}$-ray and $\mathrm{I}_{2} \mathrm{KI}+\mathrm{X}$-ray exposure. This inhibition of H\&E staining may be due to blockage of aluminium binding sites (which are utilised by Harris' Haematoxylin). The success of H\&E staining post- $\mathrm{I}_{2} \mathrm{KI}$ exposure could be due to a lack of interaction between iodine and aluminimum binding sites or the loss of iodine ions prior to H\&E staining.

Whilst it is important to show that a widely used stain such as H\&E is compatible with prior exposure to X-rays and X-ray contrast agents. This stain has low tissue specificity. The dermal ECM in human skin is composed primarily of fibrillar collagens and elastic fibres ${ }^{48}$. These supra-molecular assemblies can be specifically visualised by picrosirius red (PSR) staining and polarised light microscopy and by bright-field light microscopy of Weigert's resorcin fuchin stained tissue respectively ${ }^{49}$. Fibrillar collagens were readily identified in sections cut from X-ray exposed biopsies and the binding of PSR to basic groups in the collagen triple helix ${ }^{50}$ appeared unaffected by prior exposure to PTA or $\mathrm{I}_{2} \mathrm{KI}$ (Fig. 8A). However, the staining of elastic fibres using Weigert's (resorcin fuchin) stain which relies on the binding of iron, was again disrupted by the presence of PTA (Fig. 8B). It appears therefore that although X-ray exposure alone has little or no discernible effect on subsequent histological analysis the compatibility of specific combinations of X-ray contrast agent and histological stain may need to be established on a case-by-case basis. 
A

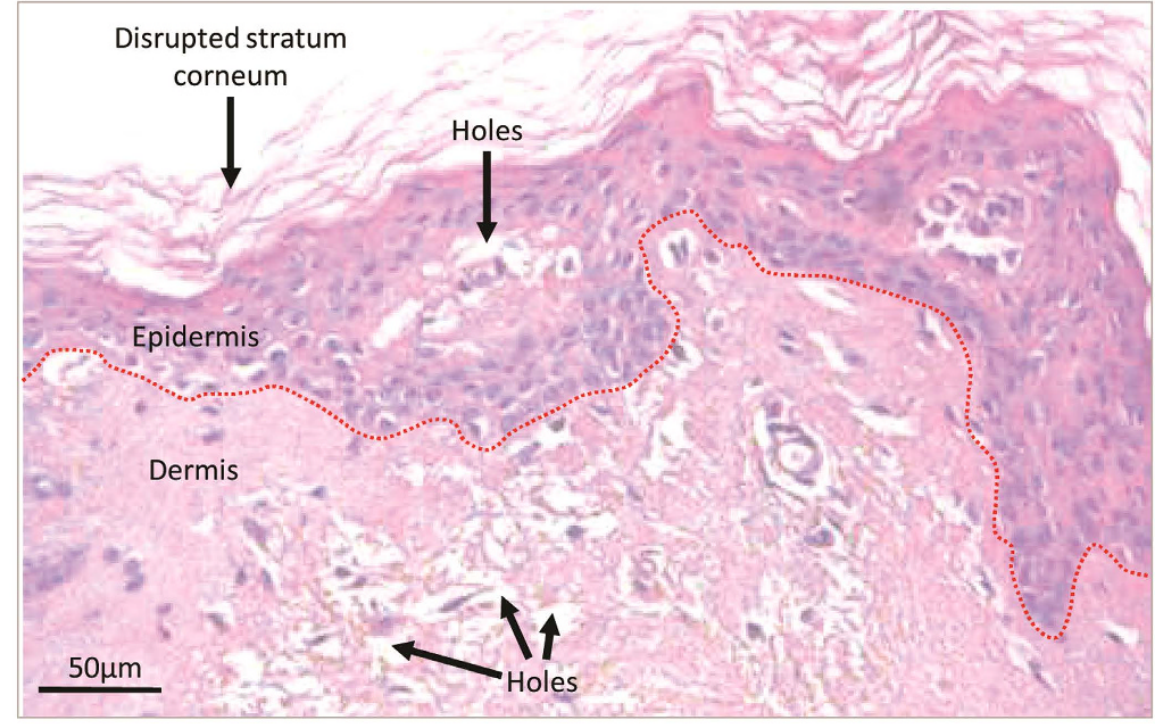

B

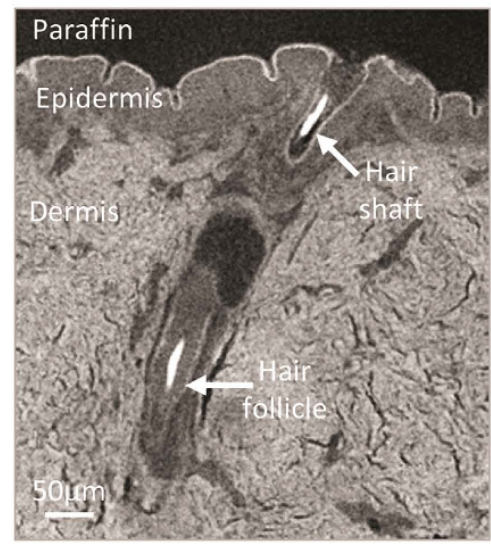

C

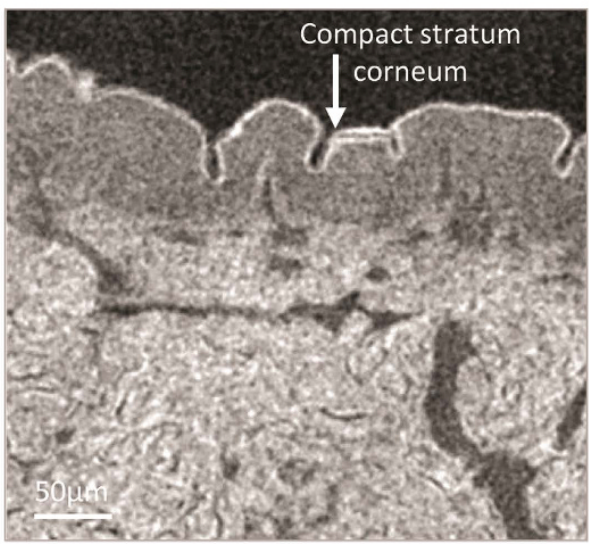

Figure 6. Sectioning induced artefacts and sub-micron X-ray tomography of human skin. (A) H\&E stained human skin biopsy. The dermal-epidermal junction is denoted by a red dotted line. (B and C) Virtual slices extracted from an X-ray tomogram of a human skin biopsy. The dermal and epidermal layers and large structures such as hair follicles are clearly visible and outer stratum corneum remains densely packed and closely adhered to the underlying tissue.

Crucially however, we also build on the observations of Chen and colleagues, who showed that microCT is compatible with immunohistochemical analyses in frog cartilage ${ }^{51}$, to demonstrate that an anti-keratin 14 antibody can successfully and specifically bind to epidermal epitopes in human skin following exposure to either X-rays alone or to both X-rays and X-ray contrast agents (Fig. 8C).

\section{Conclusions}

Here we show that major tissue structures may be resolved by sub-micron X-ray tomography of native tissues and organs and that histological and immunohistochemical analysis of tissue structure and composition is compatible with prior exposure to X-rays and some X-ray contrast agents. In the specific case of the pressurised artery we demonstrate that static intra-luminal pressure has a differential effect on the morphology and hence volume of the medial and adventitial layers.

It is clear therefore that, with little user intervention, microCT and nanoCT in combination can rapidly visualise the $3 \mathrm{D}$ structure of relatively large tissue volumes (up to $\sim 10 \mathrm{~mm}^{3}$ ) at sub- $\mu \mathrm{m}$ spatial resolutions. Compared with complementary $3 \mathrm{D}$ visualisation approaches, such as serial-sectioning combined with light or electron microscopy, or more recently developed technologies such as serial block face / SEM approaches, X-ray tomography not only maintains the same spatial resolution in all three axes but avoids artefacts induced by the loss, distortion and miss-alignment of sections ${ }^{52}$. In addition, as $\mathrm{X}$-ray tomography is non-destructive, it can be used to identify volumes of interest for subsequent complementary microscopical and analytical analysis within a correlative tomography framework ${ }^{53}$. A key 
A

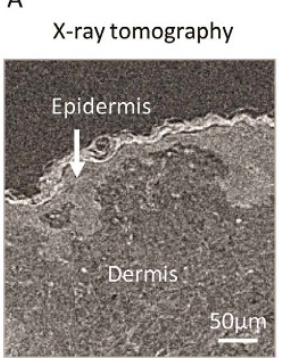

PhosphoTunstic acid (PTA) + X-ray
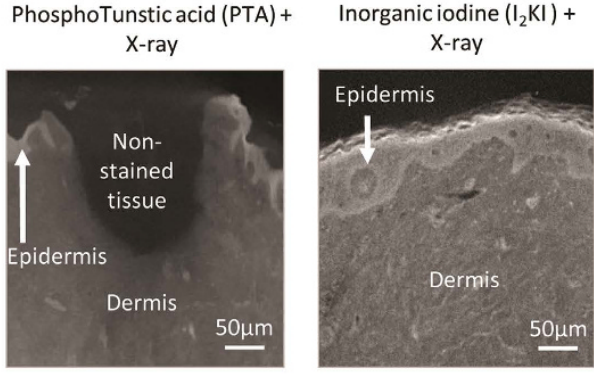

B
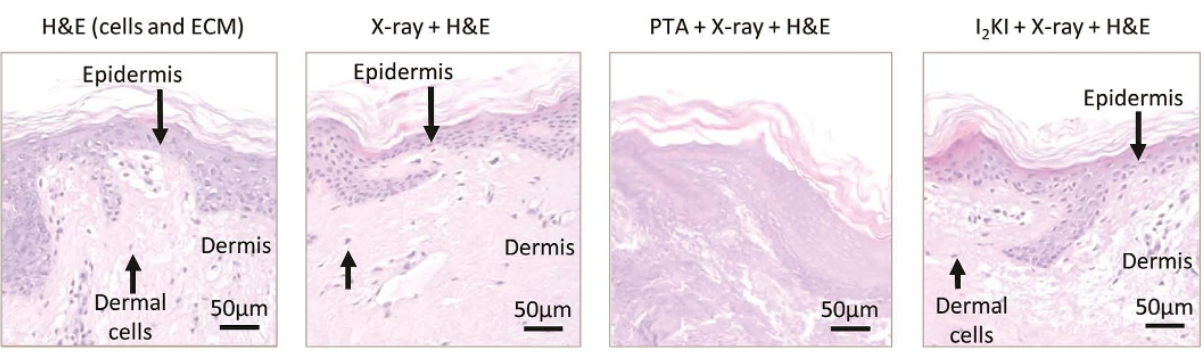

Figure 7. The use of contrast agents in the skin with X-ray tomography. Using the Carl Zeiss Versa-510 system unstained skin may be visualised. However, contrast is low and the differentiation of structures within the skin is difficult. The use of PTA or $\mathrm{I}_{2} \mathrm{KI}$ contrasting agents improves the differentiation obtained between structures in the skin. PTA provides the best contrast when looking at structures within the skin, however, due to its molecular size, its rate of staining is slow and full penetration of the tissue is not achieved, even after many hours (A). Further to this, if tissue that has been imaged using X-ray tomography is then sectioned and stained with H\&E to visualise skin structure, the expected staining pattern is disrupted by the presence of PTA. However, X-ray exposure and the use of $\mathrm{I}_{2} \mathrm{KI}$ contrasting agent does not prevent successful H\&E staining of skin sections (B).

area for improving the technique would be to image native tissues. Although, in the current study, the use of chemical fixatives and a mechanical paraffin support provide a stable medium for the collection of high quality (without movement artefacts) 3D data, chemical fixation can induce both micro- and non-structural remodelling ${ }^{54,55}$. Previously microCT has been employed to characterise short-term (milliseconds) mechanical behaviours of metals and long-term (days) developmental remodelling in living insects $^{56,57}$. It remains to be determined if microCT of native mammalian tissues is capable of resolving acute structural remodelling events in tissue sub-structure(s) as a consequence of dynamic physiological strains.

\section{Materials and Methods}

Tissue preparation, X-ray contrast agent exposure and histological and immunohistochemical staining. All procedures accorded to the UK Animals (Scientific Procedures) Act 1986 and were approved by the University of Manchester ethical review process. Pressure myography is conventionally used to study small arteries ${ }^{58-60}$. In this study we adapted the technique by using stronger sutures, larger cannulas and a surgical knotting technique to secure the rat $\mathrm{CCA}^{61}$. Left and right CCAs were dissected from 250-300g male Wistar rats (Charles River, UK). The right CCA was mounted onto a pressure myograph (CH-1-QT, Living Systems: Vermont, USA). The left CCA was chemically fixed in the unpressurised state but the right CCA was subjected to an intra-luminal pressure of $110 \mathrm{~mm} \mathrm{Hg}$ (equivalent to mean arterial pressure) ${ }^{62}$ which was maintained during fixation. All arteries (left and right CCA and descending thoracic aorta) were chemically fixed in $4 \%$ paraformaldehyde for two hours prior to ethanol dehydration and paraffin wax embedding using tygon tubing as a mould (Internal diameter $1.6 \mathrm{~mm}$, Harvard Apparatus, Kent, UK) $)^{63,64}$. The dimensions of the tubing were chosen to minimise the thickness of the external paraffin wax. Finally wax cylinders were glued onto the head of metal pins so that the vessel axis could be mounted perpendicular to the X-ray source.

The 3D structure of human skin and the effects of X-ray exposure and X-ray contrast agents on subsequent histological and immunohistochemical staining procedures were characterised using buttock skin punch biopsies (obtained with informed consent and local ethical approval from the North West Research Ethics Committee [REC] reference 07/Q1409/9) and breast skin samples donated to the Manchester Skin Health Biobank (REC reference 09/H1010/10). All experiments were carried out in accordance with the approved local REC guidelines. Following fixation in Bouin's solution, skin samples were either: i) stained with Lugol's solution $\left(\mathrm{I}_{2} \mathrm{KI}\right)$ for 16 hours followed by washing in water; ii) stained 
A Picrosirius red

(PSR: collagen)
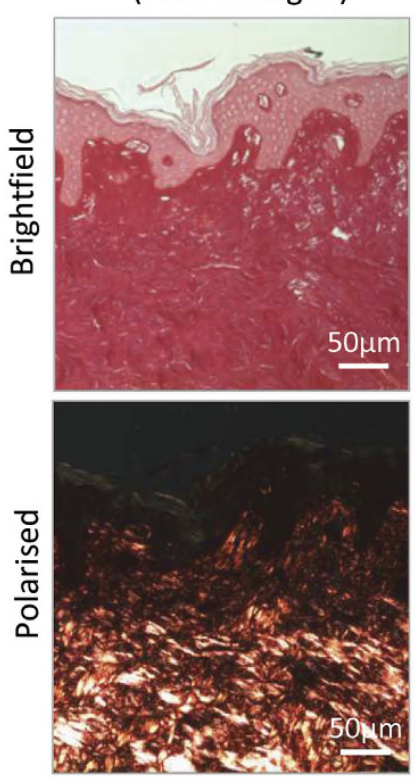

X-ray + PSR
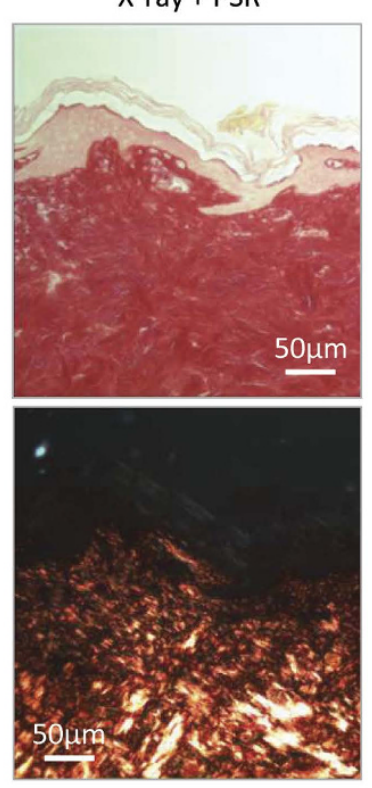

$\mathrm{PTA}+\mathrm{X}-$ ray + PSR
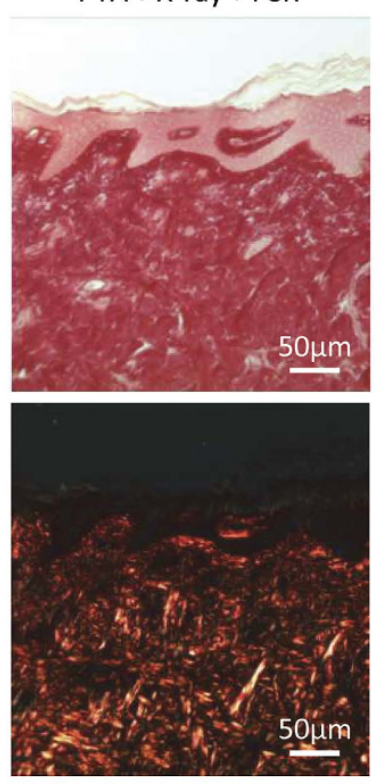

PTA + X-ray + WRF

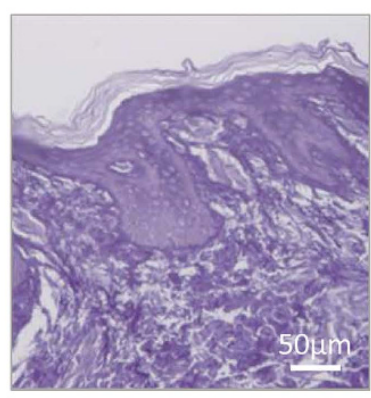

$\mathrm{PTA}+\mathrm{X}-$ ray + K14 IF

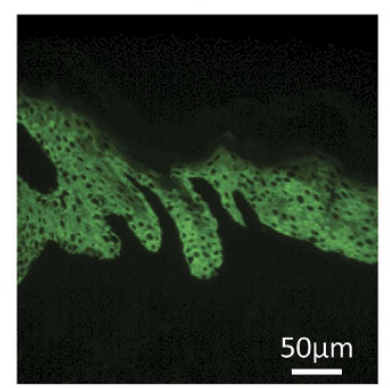

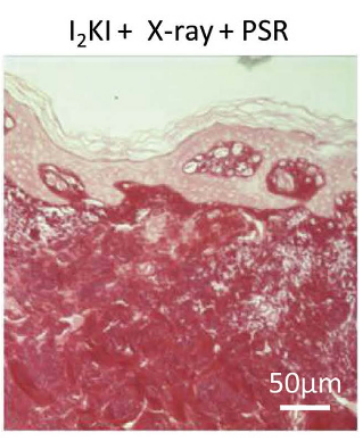

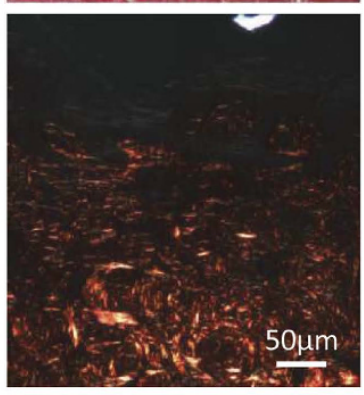

$\mathrm{I}_{2} \mathrm{KI}+\mathrm{X}$-ray + WRF

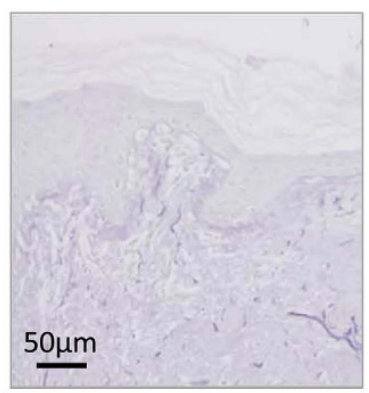

$\mathrm{I}_{2} \mathrm{KI}+\mathrm{X}$-ray $+\mathrm{K} 14 \mathrm{IF}$

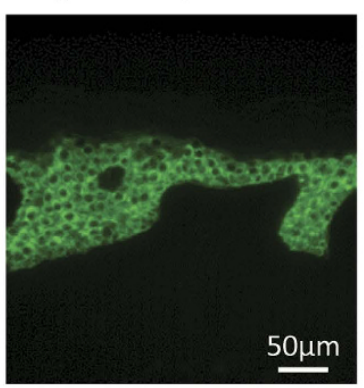

Figure 8. The effects of X-ray tomography on subsequent histology and immunofluorescence of structural components of the skin. Staining of tissue sections, from skin exposed to X-ray, for collagen show that prior staining with PTA or $\mathrm{I}_{2} \mathrm{KI}$ does not prevent the visualisation of collagen fibres (A). However, Weigert's staining of skin for elastin fibres is disrupted by the presence of PTA (B). Unlike Weigert's, exposure to X-ray and PTA or $\mathrm{I}_{2} \mathrm{KI}$ does not prevent the binding of Keratin 14 antibody to its epitope in skin, confirming that immunofluorescence is possible following X-ray tomography $(\mathbf{C})$.

with phosphotungstic acid (PTA) for 40 hours followed by washing in $70 \%$ ethanol, or iii) left unstained and submerged in $70 \%$ ethanol for 16 hours $^{5}$. 
X-ray tomography and data processing. CCAs were imaged using a Carl Zeiss Xradia Versa-510 system (Carl Zeiss: California, USA) with the X-ray source voltage and current set to $60 \mathrm{kV}$ and $83 \mathrm{~mA}$, respectively. The detector and source were positioned $\sim 35 \mathrm{~mm}$ and $\sim 10 \mathrm{~mm}$ respectively from the sample to achieve a small amount of X-ray phase contrast using the inline method with the $4 \mathrm{x}$ objective $\mathrm{e}^{30,65}$. Two successive scans were performed for each sample: i) a low resolution data collection scan of the complete artery cross-section using the $4 \mathrm{x}$ objective which achieved a voxel size of $0.75 \mu \mathrm{m}$ and; ii) a higher resolution region-of-interest scan of a smaller tissue volume which employed the 20x objective and achieved a voxel size of $0.50 \mu \mathrm{m}$ (detector to sample and sample to sources distances: $6.5 \mathrm{~mm}$ and $18 \mathrm{~mm}$ respectively). This improved resolution using the 20x objective (Fig. 2D) would be necessary to measure the dimensions of key structures such as the medial elastic lamellae. Exposure times per radiograph were in the range $6-10 \mathrm{~s}$ for the $4 \mathrm{x}$ scan, and $26-35 \mathrm{~s}$ for the $20 \mathrm{x}$ scan. Approximately 2000 projections were collected for each scan as the sample rotated over $360^{\circ}$. Further steps were taken to ensure good data quality; prior to scanning, each sample was placed within the scanner chamber overnight to ensure the sample and mount had attained thermal equilibrium $\left(28^{\circ} \mathrm{C}\right)$. Furthermore, the source was switched on for at least 30 minutes prior to each scan to improve stability.

Multiscale X-ray tomography imaging of the artery samples was carried out using Carl Zeiss Xradia Versa-510 (microCT) and Carl Zeiss Xradia Ultra-810 (nanoCT) systems. Gold beads of diameter 1-3 $\mu \mathrm{m}$ were placed on the sample to enable the same region to be identified from all scans. The aorta sample was scanned at two resolutions with the Versa XRM. The first, using the $4 \mathrm{x}$ objective, enabled the whole vessel cross-section to be imaged at $0.94 \mu \mathrm{m}$ voxel size. A second, higher resolution region-of-interest, scan was carried out using the 20x objective yielding a voxel size of $0.5 \mu \mathrm{m}$. For both scans, the source voltage was set to $60 \mathrm{kV}$ and 2001 projections were taken over $360^{\circ}$ with and exposure time of $4 \mathrm{~s}$ and $30 \mathrm{~s}$ respectively. Nano-scale imaging of the elastic lamellae and the inter-lamellar regions was then carried out using the Ultra-810. This system is an X-ray microscope based on zone plate optics and has two magnification modes giving $150 \mathrm{~nm}$ and $50 \mathrm{~nm}$ resolution respectively. The system uses X-rays with energy $5.4 \mathrm{keV}$ and can achieve Zernike phase contrast via the use of a phase ring ${ }^{35}$. A $65 \times 65 \times 65 \mu \mathrm{m}$ region-of-interest of the sample was scanned at $150 \mathrm{~nm}$ resolution using Zernike phase contrast and 1851 projections were taken over $180^{\circ}$ with an exposure time of 40 s/projection.

Buttock skin biopsies were imaged on a Carl Zeiss Xradia MicroXCT-400 system using the $4 \mathrm{x}$ objective, with a source voltage of $40 \mathrm{kV}$, a source current of $250 \mu \mathrm{A}$ and an exposure time of $30 \mathrm{~s} /$ projection. The sample was positioned $8 \mathrm{~mm}$ from the source, and $45 \mathrm{~mm}$ from the detector. X-ray contrast-enhanced $\left(\mathrm{I}_{2} \mathrm{KI}\right.$ or PTA) breast skin samples were imaged on a Carl Zeiss Xradia Versa-510 system also using the $4 \mathrm{x}$ magnification. $\mathrm{I}_{2} \mathrm{KI}$ stained tissue was imaged with 3201 projections at an exposure time of $1.5 \mathrm{~s} /$ projection, whilst PTA-stained tissue required only 601 projections at $1 \mathrm{~s} /$ projection (due to greater contrast). Following data acquisition, volumetric data was reconstructed from the scan data using the FDK algorithm ${ }^{66}$.

Segmentation and morphological characterisation of ${ }_{3} \mathrm{D}$ arterial reconstructions. Prior to segmentation, noise in the data was reduced by applying a $1 \mathrm{D}$ bilateral filter in the axial direction ${ }^{67}$. The vessel wall was then segmented in Avizo 8 using a conventional thresholding approach which relied on differences in X-ray density (Fig. 3). However, as the component medial and adventitial layers exhibited very similar X-ray densities, we developed a novel semi-automatic segmentation technique which exploited differences in grey-level texture in the two layers. Specifically, the elastic lamellae in the medial layer form concentric rings in the trans-axial plane, which become approximately straight lines when the vessel is digitally 'unwrapped'; whereas the structures in the adventitial layer are less well ordered. This segmentation protocol was applied to each trans-axial slice in the 3D data set and comprised the following stages (as depicted in Fig. 3). First, the vessel wall was unwrapped by applying a smoothing spline (fitted to the image coordinates of the lumen edge) and resampling lines perpendicular to the spline curve at equal intervals. Next, the wall was segmented from the supporting paraffin background by applying a grey level threshold. The main adventitial features (radial connection and small groups of connected pixels) were then removed by applying a $1 \mathrm{D}$ morphological opening operation along the vertical axis (Fig. 3). Finally, the boundary between the medial and adventitial layers was established. The outer internal elastic lamina was located by finding, for each row, the outer most segmented pixel (with coordinate $x e$ ). In order to deal with residual adventitial features (which can cause deviations in the boundary position) an optimisation approach was adopted, based on the fast marching method ${ }^{68,69}$, which penalises large deviations from a vertical path. This method is related to Dijktrass method for computing the shortest path in a network, and in this case was used to find the geodesic path through a weighted image. The weighted image was constructed by first determining the mean edge position along the horizontal axis, $\overline{\mathrm{x}}$ by fitting a Gaussian mixture model to the distribution of $x e+1$. Pixels with coordinates $x e+1$ that were close to $\overline{\mathrm{x}}$ were given extra weight; whereas segmented pixels from the initial thresholded image were given a lower weight. A geodesic path (which is attracted to large weights and equates to the contour followed by the medial adventitial layer) can then be extracted. This contour can then be wrapped onto the original trans-axial slices by applying the inverse of the coordinate transform found in the first step. Finally, the surface of the medial layer boundary in the 3D stack was smoothed to remove noisy features. The complete process was implemented in Matlab 2013a (Mathworks, 
Massachuesetts, USA). Some manual adjustments to the segmentation were necessary in regions where the contrast was poorer, and this was achieved using the segmentation tools in Avizo 8. Despite this need for occasional manual intervention, in general, segmentation of wall layers within a single microCT data set can be achieved within 2-3 hours.

Histological and immunohistochemical analysis. The effects of sectioning and intra-luminal pressure on the rat CCA was characterised by hematoxylin and eosin (H\&E: Sigma Aldrich, Milton Keynes, UK) staining of $5 \mu \mathrm{m}$ thick paraffin wax sections. Bright field optical images were captured using a Biozero-8000 fluorescence microscope (Keyence; Osaka, Japan). Subsequent to X-ray tomography (of both native and $\mathrm{I}_{2} \mathrm{KI}$ - or PTA- exposed), breast skin samples were sectioned, dewaxed, rehydrated and stained for cells and ECM (by H\&E; Harris' Haematoxylin, Eosin Y, Sigma Aldrich, Dorset, UK), fibrillar collagens (picrosirius red; 0.1\% Sirius red F3BA (Sigma Aldrich) in saturated aqueous picric acid for 1 hour followed by a brief rinse in $0.1 \%$ acetic acid ) and elastic fibres (Weigert's resorcin fuchsin; (Clin-Tech Ltd, Guilford, UK; 30 minutes in Weigerts and then rinsed in IMS, and then in water) ${ }^{49}$. Sections were dehydrated and mounted with DPX mounting media (Sigma-Aldrich) before imaging. Picrosirius red staining was visualised using polarised light microscopy (Leitz DMRB microscope: Leica, Milton Keynes, UK) with an Infinity X camera (DeltaPix: Maalov, Denmark), other stains were visualised using the All-in-one Type Fluorescence Microscope Biozero-8000 (Keyence; Osaka, Japan). For antigen retrieval and immunohistochemistical localisation of epidermal Keratin-14, paraffin sections were dewaxed, rehydrated, exposed to citrate buffer, permeabolised for 10 minutes at room temperature, washed in tris-buffered saline (TBS; $100 \mathrm{mmol} \mathrm{l^{-1 }}$ Tris, $150 \mathrm{mmol} \mathrm{l} \mathrm{l}^{-1} \mathrm{NaCl}$; pH 7.4) and then exposed to a rabbit anti-keratin-14 primary antibody (1/1000; clone PRB-155P; Covance, New Jersey, USA) for an hour at room temperature. Sections were then washed in TBS before the addition of 488 AlexaFluor secondary goat anti-rabbit antibody (1:500; Invitrogen, Paisley, UK) for an hour. The sections were then washed and mounted with Fluoromount ${ }^{\mathrm{TM}}$ (Sigma-Aldrich).

\section{References}

1. Rastogi, V. et al. Artefacts: a diagnostic dilemma - a review. Journal of clinical and diagnostic research: JCDR 7, 2408-2413, doi:10.7860/jcdr/2013/6170.3541 (2013).

2. Fujiwara, T. \& Uehara, Y. The cytoarchitecture of the medial layer in rat thoracic aorta - a scanning electron-microscopic study. Cell and Tissue Research 270, 165-172, doi:10.1007/bf00381891 (1992).

3. O'Connell, M. K. et al. The three-dimensional micro- and nanostructure of the aortic medial lamellar unit measured using 3D confocal and electron microscopy imaging. Matrix Biol. 27, 171-181, doi:10.1016/j.matbio.2007.10.008 (2008)

4. Schrauwen, J. T. C. et al. A method for the quantification of the pressure dependent $3 \mathrm{D}$ collagen configuration in the arterial adventitia. J. Struct. Biol. 180, 335-342, doi:10.1016/j.jsb.2012.06.007 (2012).

5. Metscher, B. D. MicroCT for Developmental Biology: A Versatile Tool for High-Contrast 3D Imaging at Histological Resolutions. Developmental Dynamics 238, 632-640, doi:10.1002/dvdy.21857 (2009).

6. Mizutani, R. \& Suzuki, Y. X-ray microtomography in biology. Micron 43, 104-115, doi:10.1016/j.micron.2011.10.002 (2012).

7. Mitchell, G. F. Effects of central arterial aging on the structure and function of the peripheral vasculature: implications for endorgan damage. Journal of Applied Physiology 105, 1652-1660, doi:10.1152/japplphysiol.90549.2008 (2008).

8. Wagenseil, J. E. \& Mecham, R. P. Vascular Extracellular Matrix and Arterial Mechanics. Physiol. Rev. 89, 957-989, doi:10.1152/ physrev.00041.2008 (2009).

9. Cruickshank, K. et al. Aortic pulse-wave velocity and its relationship to mortality in diabetes and glucose intolerance - An integrated index of vascular function? Circulation 106, 2085-2090, doi:10.1161/01.cir.0000033824.02722.f7 (2002).

10. Judge, D. P. \& Dietz, H. C. Marfan's syndrome. Lancet 366, 1965-1976, doi:10.1016/s0140-6736(05)67789-6 (2005).

11. Tsamis, A., Krawiec, J. T. \& Vorp, D. A. Elastin and collagen fibre microstructure of the human aorta in ageing and disease: a review. J. R. Soc. Interface 10, doi:10.1098/rsif.2012.1004 (2013).

12. Wolinsky, H. \& Glagov, S. Structural bassis for static and mechanical properties of aortic media. Circ.Res. 14, 400-\& (1964).

13. Carroll, J. F., Huang, M., Hester, R. L., Cockrell, K. \& Mizelle, H. L. Hemodynamic alterations in hypertensive obese rabbits. Hypertension 26, 465-470 (1995)

14. Gasser, T. C., Ogden, R. W. \& Holzapfel, G. A. Hyperelastic modelling of arterial layers with distributed collagen fibre orientations. J. R. Soc. Interface 3, 15-35, doi:10.1098/rsif.2005.0073 (2006).

15. Humphrey, J. D. \& Na, S. Elastodynamics and arterial wall stress. Ann. Biomed. Eng. 30, 509-523, doi:10.1114/1.1467676 (2002).

16. Dingemans, K. P., Teeling, P., Lagendijk, J. H. \& Becker, A. E. Extracellular matrix of the human aortic media: An ultrastructural histochemical and immunohistochemical study of the adult aortic media. Anat. Rec. 258, 1-14 (2000).

17. Schriefl, A. J., Wolinski, H., Regitnig, P., Kohlwein, S. D. \& Holzapfel, G. A. An automated approach for three-dimensional quantification of fibrillar structures in optically cleared soft biological tissues. J. R. Soc. Interface 10, doi:10.1098/rsif.2012.0760 (2013).

18. Huijsmans, C. J., Damen, J., van der Linden, J. C., Savelkoul, P. H. \& Hermans, M. H. Comparative analysis of four methods to extract DNA from paraffin-embedded tissues: effect on downstream molecular applications. BMC research notes 3, 239, doi:10.1186/1756-0500-3-239 (2010).

19. Verdonck, M. et al. Breast cancer and melanoma cell line identification by FTIR imaging after formalin-fixation and paraffinembedding. Analyst 138, 4083-4091, doi:10.1039/c3an00246b (2013).

20. Slager, C. J. et al. The role of shear stress in the generation of rupture-prone vulnerable plaques. Nature Clinical Practice Cardiovascular Medicine 2, 401-407, doi:10.1038/ncpcardio0274 (2005).

21. Li, X., Yang, Q., Wang, Z. \& Wei, D. Shear Stress in Atherosclerotic Plaque Determination. DNA and cell biology, doi:10.1089/ dna.2014.2480 (2014)

22. Aslanidi, O. V. et al. Application of Micro-Computed Tomography With Iodine Staining to Cardiac Imaging, Segmentation, and Computational Model Development. IEEE Trans. Med. Imaging 32, 8-17, doi:10.1109/tmi.2012.2209183 (2013).

23. Butters, T. D. et al. Optimal Iodine Staining of Cardiac Tissue for X-Ray Computed Tomography. Plos One 9, doi:10.1371/journal. pone.0105552 (2014).

24. Horng, A. et al. Cartilage and Soft Tissue Imaging Using X-rays Propagation-Based Phase-Contrast Computed Tomography of the Human Knee in Comparison With Clinical Imaging Techniques and Histology. Investigative Radiology 49, 627-634 (2014). 
25. Kalson, N. S., Malone, P. S. C., Bradley, R. S., Withers, P. J. \& Lees, V. C. Fibre bundles in the human extensor carpi ulnaris tendon are arranged in a spiral. Journal of Hand Surgery-European Volume 37E, 550-554, doi:10.1177/1753193411433228 (2012).

26. Holme, M. N. et al. Complementary X-ray tomography techniques for histology-validated 3D imaging of soft and hard tissues using plaque-containing blood vessels as examples. Nat. Protoc. 9, 1401-1415, doi:10.1038/nprot.2014.091 (2014).

27. Pai, V. M. et al. Coronary artery wall imaging in mice using osmium tetroxide and micro-computed tomography (micro-CT). J. Anat. 220, 514-524, doi:10.1111/j.1469-7580.2012.01483.x (2012).

28. Naveh, G. R. S., Brumfeld, V., Dean, M., Shahar, R. \& Weiner, S. Direct MicroCT imaging of non-mineralized connective tissues at high resolution. Connect. Tissue Res. 55, 52-60, doi:10.3109/03008207.2013.867333 (2014).

29. Bravin, A., Coan, P. \& Suortti, P. X-ray phase-contrast imaging: from pre-clinical applications towards clinics. Physics in Medicine and Biology 58, R1-R35, doi:10.1088/0031-9155/58/1/r1 (2013).

30. Bradley, R. S., McNeil, A. \& Withers, P. J. An examination of phase retrieval algorithms as applied to phase contrast tomography using laboratory sources. Developments in X-Ray Tomography Vii 7804, doi:10.1117/12.860536 (2010).

31. International, C. Z. Contrast with Submicron 3D X-ray Imaging For Difficult-to-Image Materials. (2014).

32. Incorporated, P. E. XRD1621NES <http://www.perkinelmer.co.uk/PDFs/downloads/DTS_16inchDigitalXrayDetectors.pdf>

33. Graham, H. K. et al. Tissue section AFM: In situ ultrastructural imaging of native biomolecules. Matrix Biol. 29, 254-260, doi:10.1016/j.matbio.2010.01.008 (2010).

34. Withers, P. J. X-ray nanotomography. Mater. Today 10, 26-34, doi:10.1016/s1369-7021(07)70305-x (2007).

35. Tkachuk, A. et al. X-ray computed tomography in Zernike phase contrast mode at $8 \mathrm{keV}$ with $50-\mathrm{nm}$ resolution using $\mathrm{Cu}$ rotating anode X-ray source. Z. Kristall. 222, 650-655, doi:10.1524/zkri.2007.222.11.650 (2007).

36. Mokso, R. et al. X-ray mosaic nanotomography of large microorganisms. J. Struct. Biol. 177, 233-238, doi:10.1016/j.jsb.2011.12.014 (2012).

37. Andrews, J. C. et al. Nanoscale X-Ray Microscopic Imaging of Mammalian Mineralized Tissue. Microsc. microanal. 16, 327-336, doi:10.1017/s1431927610000231 (2010).

38. Reed, T. R. \& Dubuf, J. M. H. A Review of Recent Texture Segmentation and Feature Extraction Techniques. CVGIP: Image Understanding 57, 359-372, doi:http://dx.doi.org/10.1006/ciun.1993.1024 (1993).

39. Lesage, D., Angelini, E. D., Bloch, I. \& Funka-Lea, G. A review of 3D vessel lumen segmentation techniques: Models, features and extraction schemes. Med. Image Anal. 13, 819-845, doi:10.1016/j.media.2009.07.011 (2009).

40. Ruskó, L., Bekes, G. \& Fidrich, M. Automatic segmentation of the liver from multi- and single-phase contrast-enhanced CT images. Med. Image Anal. 13, 871-882, doi:http://dx.doi.org/10.1016/j.media.2009.07.009 (2009).

41. Xiong, G. L., Taylor, C. A. \& Ieee. Influence of vessel roughness on wall shear stress in image-based blood flow moedling. 2010 7th Ieee International Symposium on Biomedical Imaging: From Nano to Macro, 33-36 (2010).

42. Nolan, D. R., Gower, A. L., Destrade, M., Ogden, R. W. \& McGarry, J. P. A robust anisotropic hyperelastic formulation for the modelling of soft tissue. Journal of the mechanical behavior of biomedical materials 39, 48-60, doi:10.1016/j.jmbbm.2014.06.016 (2014).

43. Sherratt, M. J. Age-Related Tissue Stiffening: Cause and Effect. Advances in Wound Care 2, 11-17, doi:10.1089/wound.2011.0328 (2012).

44. Cecchetto, G. et al. Estimation of the firing distance through micro-CT analysis of gunshot wounds. Int. J. Legal Med. 125, 245-251, doi:10.1007/s00414-010-0533-6 (2011).

45. Suh, J. W. et al. in Medical Image Computing and Computer-Assisted Intervention - Miccai 2009, Pt I, Proceedings Vol. 5761 Lecture Notes in Computer Science (eds G. Z. Yang et al.) 688-695 (Springer-Verlag, Berlin, 2009).

46. Handschuh, S., Baeumler, N., Schwaha, T. \& Ruthensteiner, B. A correlative approach for combining microCT, light and transmission electron microscopy in a single 3D scenario. Front. Zool. 10, 1-16 (2013).

47. de la Cuesta, F. B. et al. Collagen imaged by Coherent X-ray Diffraction: towards a complementary tool to conventional scanning SAXS. Xiv International Conference on Small-Angle Scattering (Sas09) 247, doi:10.1088/1742-6596/247/1/012004 (2010).

48. Naylor, E. C., Watson, R. E. B. \& Sherratt, M. J. Molecular aspelts of skin ageing. Maturitas 69, 249-256 (2011)

49. Graham, H. K. et al. Localised micro-mechanical stiffening in the ageing aorta. Mech. Ageing Dev. 132, 459-467, doi:10.1016/j. $\operatorname{mad} .2011 .07 .003$ (2011).

50. Junqueira, L. C. U., Bignolas, G. \& Brentani, R. R. Picrosirius staining plus polarizarion miroscopy, a specific method for collagen detection in tissue sections. Histochem.J. 11, 447-455, doi:10.1007/bf01002772 (1979).

51. Chen, Y., Lin, G. F., Chen, Y. C., Fok, A. \& Slack, J. M. W. Micro-Computed Tomography for Visualizing Limb Skeletal Regeneration in Young Xenopus Frogs. Anatomical Record-Advances in Isntegrative Anatomy and Evolutionary Biology 295, 1562-1565, doi:10.1002/ar.22496 (2012).

52. Denk, W. \& Horstmann, H. Serial block-face scanning electron microscopy to reconstruct three-dimensional tissue nanostructure. PLoS. Biol. 2, 1900-1909, doi:10.1371/journal.pbio.0020329 (2004).

53. Burnett, T. L. et al. Correlative Tomography. Sci Rep 4, doi:10.1038/srep04711 (2014).

54. Viidik, A. \& Lewin, T. Changes in tensile strength characteristics and histology of rabbit ligsaments induced by different modes of postmortal storage. Acta Orthop. 37, 141-155 (1966).

55. Tilley, J. M. R., Carr, A. J. \& Czernuszka, J. T. Atomic Force Microscopy of bulk tendon samples: Affect of location and fixation on tissue ultrastructure. Micron 42, 531-535, doi:10.1016/j.micron.2011.01.001 (2011).

56. Walker, S. M. et al. In Vivo Time-Resolved Microtomography Reveals the Mechanics of the Blowfly Flight Motor. PLoS. Biol. 12, doi:10.1371/journal.pbio.1001823 (2014).

57. Lowe, T., Garwood, R. J., Simonsen, T. J., Bradley, R. S. \& Withers, P. J. Metamorphosis revealed: time-lapse three-dimensional imaging inside a living chrysalis. J. R. Soc. Interface 10, doi:10.1098/rsif.2013.0304 (2013).

58. Schiffrin, E. L. \& Hayoz, D. How to assess vascular remodelling in small and medium-sized muscular arteries in humans. J. Hypertens. 15, 571-584, doi:10.1097/00004872-199715060-00002 (1997).

59. Hausman, N., Martin, J., Taggart, M. J. \& Austin, C. Age-related changes in the contractile and passive arterial properties of murine mesenteric small arteries are altered by caveolin-1 knockout. J. Cell. Mol. Med. 16, 1720-1730, doi:10.1111/j.1582-4934.2011.01457.x (2012).

60. Schofield, I., Malik, R., Izzard, A., Austin, C. \& Heagerty, A. Vascular structural and functional changes in type 2 diabetes mellitus - Evidence for the roles of abnormal myogenic responsiveness and dyslipidemia. Circulation 106, 3037-3043, doi:10.1161/01. cir.0000041432.80615.a5 (2002).

61. Faury, G. et al. Developmental adaptation of the mouse cardiovascular system to elastin haploinsuffliciency. J. Clin. Invest. 112, 1419-1428, doi:10.1172/jci200319028 (2003).

62. Borges, J. P., Masson, G. S., Tibirica, E. \& Lessa, M. A. Aerobic Interval Exercise Training Induces Greater Reduction in Cardiac Workload in the Recovery Period in Rats. Arq. Bras. Cardiol. 102, 47-52, doi:10.5935/abc.20130230 (2014).

63. Sweeney, M., Jones, C. J. P., Greenwood, S. L., Baker, P. N. \& Taggart, M. J. Ultrastructural features of smooth muscle and endothelial cells of isolated isobaric human placental and maternal arteries. Placenta 27, 635-647, doi:10.1016/j. placenta.2005.05.010 (2006). 
64. Carta, L. et al. Fibrillins 1 and 2 perform partially overlapping functions during aortic development. J. Biol. Chem. 281, 80168023, doi:10.1074/jbc.M511599200 (2006).

65. Wilkins, S. W., Gureyev, T. E., Gao, D., Pogany, A. \& Stevenson, A. W. Phase-contrast imaging using polychromatic hard X-rays. Nature 384, 335-338, doi:10.1038/384335a0 (1996).

66. Kak, A. C. \& Slaney, M. Principles of Computerized Tomographic Imaging. (IEEE Press, 1988).

67. Tomasi, C., Manduchi, R. \& Ieee. Bilateral filtering for gray and color images. (Narosa Publishing House, 1998).

68. Sethian, J. A. A fast marching level set method for monotonically advancing fronts. Proc. Natl. Acad. Sci. USA 93, 1591-1595, doi:10.1073/pnas.93.4.1591 (1996).

69. Schena, G. \& Favretto, S. Pore space network characterization with sub-voxel definition. Transp. Porous Media 70, 181-190, doi:10.1007/s11242-006-9094-z (2007).

\section{Acknowledgements}

The authors are grateful to the staff of the Manchester X-ray Imaging Facility and the wish to acknowledge the insightful histological advice of Dr Peter Walker (University of Manchester Faculty of Life Sciences Histology Core Facility). MJS and CA gratefully acknowledge The University of Manchester and the Biotechnology and Biological Sciences Research Council (www.bbsrc.ac.uk) for funding a studentship awarded to LAW. REBW and MJS wish to acknowledge funding by DSM Nutritional Products Ltd. (www.dsm.com) and by the Medical Research Council (www.mrc.ac.uk: grant G1001398). MicroCT and nanoCT imaging was conducted at the Manchester X-ray Imaging Facility which was funded in part by the EPSRC (www.epsrc.ac.uk: grants EP/F007906/1, EP/F001452/1 and EP/I02249X/1). RSB is funded in part by the Zeiss XRM Fellowship programme.

\section{Author Contributions}

L.A.W., R.S.B, C.A., V.L.N. and M.J.S. designed the study. L.A.W., R.S.B. and V.L.N. performed the experiments and analysis with additional input from M.J.S. R.S.B., L.A.W., V.L.N. and M.J.S. prepared the figures. All authors contributed to writing and reviewing the manuscript.

\section{Additional Information}

Supplementary information accompanies this paper at http://www.nature.com/srep

Competing financial interests: The authors declare no competing financial interests.

How to cite this article: Walton, L. A. et al. Morphological Characterisation of Unstained and Intact Tissue Micro-architecture by X-ray Computed Micro- and Nano-Tomography. Sci. Rep. 5, 10074; doi: 10.1038/srep10074 (2015).

(c) (i) This work is licensed under a Creative Commons Attribution 4.0 International License. The images or other third party material in this article are included in the article's Creative Commons license, unless indicated otherwise in the credit line; if the material is not included under the Creative Commons license, users will need to obtain permission from the license holder to reproduce the material. To view a copy of this license, visit http://creativecommons.org/licenses/by/4.0/ 